CORRECTION

https://doi.org/10.1038/s41586-018-0355-0

\title{
Publisher Correction: A naturally occurring antiviral ribonucleotide encoded by the human genome
}

Anthony S. Gizzi, Tyler L. Grove, Jamie J. Arnold, Joyce Jose, Rohit K. Jangra, Scott J. Garforth, Quan Du, Sean M. Cahill, Natalya G. Dulyaninova, James D. Love, Kartik Chandran, Anne R. Bresnick, Craig E. Cameron \& Steven C. Almo

Correction to: Nature https://doi.org/10.1038/s41586-018-0238-4, published online 20 June 2018.

In the HTML version of this Letter, owing to a typesetter error, Extended Data Fig. 4 incorrectly corresponded to Fig. 4 (the PDF version of the figure was correct). This has been corrected online. 